\title{
BAO Plate Archive digitization, creation of electronic database and its scientific usage
}

\author{
A. M. Mickaelian, H. V. Abrahamyan, H. R. Andreasyan, \\ N. M. Azatyan, S. V. Farmanyan, K. S. Gigoyan, \\ M. V. Gyulzadyan, K. G. Khachatryan, A. V. Knyazyan, \\ G. R. Kostandyan, G. A. Mikayelyan, E. H. Nikoghosyan, \\ G. M. Paronyan and A. V. Vardanyan
}

Byurakan Astrophysical Observatory (BAO), Byurakan 0213, Aragatzotn Province, Armenia email: aregmick@yahoo.com

\begin{abstract}
Astronomical plate archives created on the basis of numerous observations at many observatories are important part of the astronomical heritage. Byurakan Astrophysical Observatory (BAO) plate archive consists of 37,000 photographic plates and films, obtained at $2.6 \mathrm{~m}$ telescope, $1 \mathrm{~m}$ and $0.5 \mathrm{~m}$ Schmidt telescopes and other smaller ones during 1947-1991. In 20022005, the famous Markarian Survey (First Byurakan Survey, FBS) 2000 plates were digitized and the Digitized FBS (DFBS) was created. New science projects have been conducted based on these low-dispersion spectroscopic material. In 2015, we have started a project on the whole BAO Plate Archive digitization, creation of electronic database and its scientific usage. The project will run during 3 years in 2015-2017 and the final result will be an electronic database and online interactive sky map to be used for further research projects.
\end{abstract}

Keywords. telescopes, instrumentation: miscellaneous, methods: data analysis, techniques: image processing, techniques: spectroscopic, standards, astronomical data bases: miscellaneous

\section{Introduction}

A project on Digitization of Byurakan Astrophysical Observatory (BAO) Plate Archive and creation of BAO Interactive Astronomical Database (shortly BAO Plate Archive project, BAO PAP) has started in 2015. It is aimed at preservation of BAO valuable observational material accumulated during 1947-1991, creation of full Database of all BAO observations, creation of BAO Interactive Sky Map with visualization of all observations and quick access to the data, development and accomplishment of new research projects based on the existing observational material, and integration of $\mathrm{BAO}$ observations into the international databases. A number of BAO young astronomers are involved in this project and it will last 3 years.

BAO Plate Archive is one of the largest astronomical archives in the world and is considered to be BAO main observational treasure. Today BAO archive holds some 37,000 astronomical plates, films or other carriers of observational data.

\section{BAO telescopes and observing programmes}

BAO observers worked with a number of BAO telescopes during 1947-1991 and obtained several dozens of thousands plates, films and other products. The table gives general understanding on observations of 10 BAO telescopes that worked on photographic photometry, electrophotometry, slit and objective prism spectroscopy, and polarimetry of many thousands astronomical objects. We give in Table 1 an overview of BAO telescopes 
Table 1. Overview of BAO telescopes and produced observational material.

\begin{tabular}{|c|c|c|c|c|}
\hline Telescope & Sizes $[\mathrm{cm}]$ & Years & | Observing methods | & | Plates $\mid$ \\
\hline 5" double-astrograph & 13 & $1947-1950$ & photometry & 3000 \\
\hline & 15 & $1947-1950$ & photometry & 3000 \\
\hline $8 "$ Schmidt & $20 / 20 / 31$ & $1949-1968$ & photometry & 4500 \\
\hline 20" Cassegrain & $51 / 800$ & $1952-1991$ & electrophotometry & \\
\hline 10" telescope-spectrograph & 25 & 1953-19?? & spectra & \\
\hline Nebular spectrograph & & $1954-19 ? ?$ & spectra & \\
\hline 16" Cassegrain & $41 / 400$ & $1955-1991$ & electrophotometry & \\
\hline $21 "$ Schmidt & $53 / 53 / 183$ & $1955-1991$ & photometry & 12000 \\
\hline 40" Schmidt (AZT-10) & $102 / 132 / 213$ & $1960-1991$ & photometry, spectra & 7500 \\
\hline $\mathrm{ZTA}-2.6 \mathrm{~m}$ & $264 / 1016$ & $1975-1991$ & photometry, spectra & 7000 \\
\hline All telescopes & & $1947-1991$ & & 37000 \\
\hline
\end{tabular}

and produced observational material. Telescope "Sizes" are given for the mirror and focal length for classical telescopes and for the correcting lens, mirror and focal length for Schmidt type telescopes.

Main observational projects run at three most important BAO telescopes were: 21" (0.5m) Schmidt (Polarization of cometary nebula NGC 2261, Nuclei of nearby Sa and Sb galaxies, Nuclei of nearby Sc galaxies, Search for flare stars in Pleiades, Orion, NGC 7000 (Cygnus), Praesepe and Taurus Dark Clouds (TDC), Variability of Markarian galaxies, Monitoring of extragalactic supernovae in certain areas, etc.), 40" (1m) Schmidt: (Detailed colorimetry of bright galaxies, First Byurakan Survey (FBS, Markarian survey; Markarian 1989), Search for flare stars in Pleiades, Orion, NGC 7000 (Cygnus), Praesepe and Taurus Dark Clouds (TDC), Second Byurakan Survey (SBS; Stepanian 2005), Extension of the FBS in the Galactic Plane, etc.) and ZTA-2.6m telescope: (Morphological study of Markarian galaxies, Investigation of star clusters, Investigation of groups and clusters of galaxies, Spectroscopy FBS blue stellar objects, FBS late-type stars, SBS galaxies and stellar objects (BAO/SAO), T Tauri and flare stars, ByurakanIRAS Galaxies (BIG objects) and ROSAT AGN candidates (BAO/HS/OHP/INAOE), and Direct images of the central regions of Markarian galaxies).

Especially efficient were Byurakan surveys accomplished by Markarian and colleagues.

\section{BAO Plate Archive Project}

The digitization of astronomical plates and films pursues not only the maintenance task, but also it will serve as a source for new scientific research and discoveries, if only the digitized material runs according to modern standards and, due to its accessibility, it will become an active archive. The project is aimed at compilation, accounting, digitization of BAO observational archive photographic plates and films, as well as their incorporation in databases with modern standards and methods, providing access for all observational material and development of new scientific programs based on this material.

Scientific Programs Board (SPB) is created to evaluate the existing observational material, to select sets of priorities to be scanned first and to propose new research projects. It consists of BAO Director and most experienced BAO observers, as well as researchers from NAS RA Institute of Informatics and Automation Problems (IIAP) are involved for their experience in computer science related to databases and computational methods. Project Executing Team (PET) consists of 14 members. The scanning will be carried out with 2 EPSON Perfection V750 Pro scanners.

The project consists of the following works: Creation of the Project Database, Project Webpage and User Interface, Scanning of photographic plates and films, Astrometric solution, Extraction of images and spectra, Wavelength calibration (for spectra), Density 


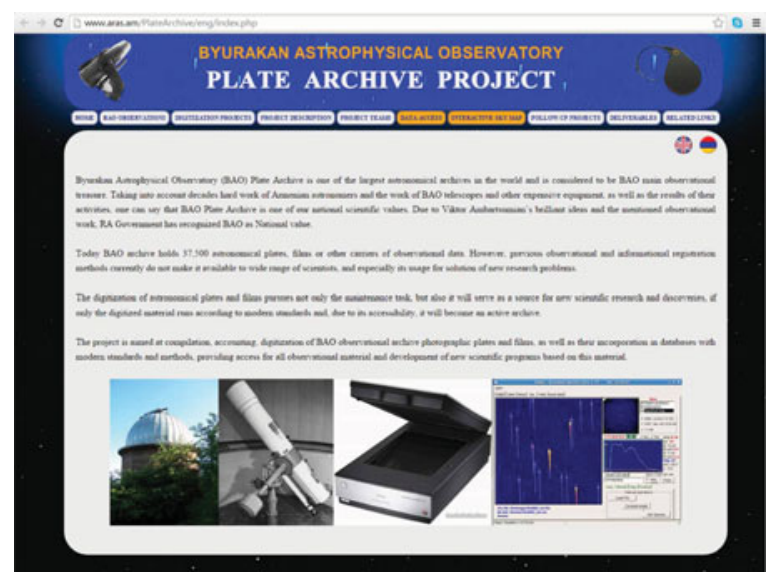

Figure 1. BAO Plate Archive Project webpage.

and flux calibration, Making up template low-dispersion spectra, Numerical classification of spectra, Visualization of BAO observations on sky map, Creation of electronic interactive sky map and search system, Scientific analysis of existing observational material and providing new research possibilities, and Proposing and discussing new research projects.

BAO PAP webpage (http://www.aras.am/PlateArchive/) was recently open and contains information on BAO observations, previous digitization projects, present Project details, teams, follow-up research projects, deliverables and related links (many items will be filled in during the next months). However, the main products will be "Data Access" and "Interactive Sky Map". The first one will contain BAO Observational Database, Search by any parameter, Data Visualization and Download of the digitized plates, films, part of them or individual objects images or spectra. "Interactive Sky Map" will visualize the observed by BAO telescopes sky and will give possibility to check observed areas for a given observational project, given telescope, observer, observing method, limiting magnitude, etc. There will be possibility to check individual fields for presence and number of plates to propose further research projects.

\section{Previous digitization programmes at $\mathrm{BAO}$ and research projects}

A number of digitization projects have been accomplished at BAO, including the most important one, Digitized First Byurakan Survey (DFBS; Mickaelian et al. 2007) based on the digitization of the famous Markarian Survey (Markarian et al. 1989). Pixel size is $15.875 \mu \mathrm{m}$ or 1.542 ", each plate is $9601 \times 9601$ pixels and each plate is $180 \mathrm{MB}$ file. Each low-dispersion spectrum is $107 \times 5$ pixels $(1700 \mu \mathrm{m}$ length). Astrometric solution has $\sim 1$ " rms. Average dispersion is $33 \AA /$ pix (22-60 from blue to red part). Photometric accuracy is $\sim 0.3^{m}$. In total there are 1874 digitized plates and the database is $\sim 400 \mathrm{~GB}$. There are 40,000,000 spectra for 20,000,000 objects. DFBS plate database is available in Vizier, Strasbourg (Mickaelian et al. 2005). The spectra extraction and analysis software is described in Mickaelian et al.(2010) and Knyazyan et al. (2011).

The Second Byurakan Survey (SBS; Stepanian 2005) plates are also subject for digitization, as they are hypersensitized and their emulsion is more sensitive for deterioration. 180 plates have been digitized so far. Due to SBS smaller photographic grains, 2400 dpi (10 $\mu \mathrm{m}$ pixel size) is being used and $512 \mathrm{MB}$ files are being obtained for each plate.

Photographic spectra of the FBS blue stellar objects (BSOs) have been obtained using $2.6 \mathrm{~m}$ telescope and UAGS spectrograph on photographic films. $\sim 700$ such spectra have been scanned with $1600 \mathrm{dpi}, 16$ bit and $650 \times 21$ pix sizes images were obtained (FBS 
BSOs; Mickaelian 2008 \& late-type stars; Gigoyan \& Mickaelian 2012). All spectra were put in a standard format, so that automatic reduction was possible. 101 FBS blue stellar objects were published and a number of planetary nebulae, white dwarfs, hot subdwarfs and HBB stars have been revealed (Sinamyan \& Mickaelian 2009).

Another project was the study of long-term variability of ON 231, which appeared in the Coma field, where photographic chains for discovery of flare stars were carried out. In total 189 plates with a total number of more than 1200 exposures in 1969-1976 with the Byurakan 21" and 40" Schmidt telescopes were obtained. This was a valuable material for study of ON231 long-term variability (Erastova 2004).

\section{Summary}

BAO Plate Archive Project will lead to preservation of BAO valuable observational material obtained during 1947-1991. However, our goal is not only to create a passive archive of scanned plates and films, but also to make use of especially those fields, where more studies are possible. Proper motion and variability studies are most important, as time domain material is contained in historical plates.

There are a number of further possible research projects that will be conducted having the plates digitized, such as Correction of ephemerides of known asteroids and search for new asteroids (Berthier et al. 2009), Discovery and study of variable stars, Revealing high proper motion stars, Study of variability of known blazars and discovery of new blazars, Revealing Novae and Supernovae progenitors, Discovery of new QSOs, new white dwarfs, new late-type stars (Gigoyan et al. 2010) and optical sources of gamma-ray bursts, Optical identifications of X-ray, IR and radio sources (Mickaelian \& Sargsyan 2004; Mickaelian \& Gigoyan 2006; Mickaelian et al. 2006; Hovhannisyan et al. 2009).

Armenian Virtual Observatory (ArVO) was created in 2005 to maintain and actively use DFBS database, and BAO Plate Archive Project will enrich it with many more images and spectra that are subject for further studies together with other available multi-wavelength and multi-time-domain data (Mickaelian et al. 2010).

\section{References}

Berthier J., Sarkissian A., Mickaelian A., \& Thuillot W. 2009, EPSC, 4, 526

Erastova, L. K. 2004, AधAT, 23, 209

Gigoyan K. S. \& Mickaelian A. M. 2012, MNRAS, 419, 3346

Gigoyan K. S., Sinamyan P. K., Engels D., \& Mickaelian A. M. 2010, Astrophysics, 53, 123

Hovhannisyan L. R., Weedman D. W., Mickaelian A. M., et al. 2009, AJ, 138, 251

Knyazyan A., Mickaelian A. M., \& Astsatryan H. 2011, Int. J. "Inf. Theories and Appl.", 18, 243

Markarian, B. E., Lipovetsky, V. A., Stepanian, J. A., et al. 1989, Comm. SAO, 62, 5

Mickaelian, A. M. 2008, AJ, 136, 946

Mickaelian, A. M. \& Gigoyan, K. S. 2006, A\& A, 455, 765

Mickaelian, A. M., Hagen, H.-J., Sargsyan, L. A., \& Mikayelyan, G. A. 2005, Cat. VI/116 at $C D S$

Mickaelian, A. M., Hovhannisyan, L. R., Engels, D., et al. 2006, A\&A, 449, 425

Mickaelian, A. M., Nesci, R., Rossi C., et al. 2007, A\&A, 464, 1177

Mickaelian, A. M. \& Sargsyan, L. A. 2004, Astrophysics, 47, 213

Mickaelian, A. M., Sargsyan, L. A., \& Mikayelyan, G. A. 2010, Proc. of Science, 30

Mickaelian, A. M., Sargsyan, L. A., Nesci, R., et al. 2010, ASP CS, Vol. 434: ADASS XIX, p. 325

Sinamyan, P. K. \& Mickaelian, A. M. 2009, Astrophysics, 52, 76

Stepanian, J. A. 2005, RMxAA, 41, 155 